
\title{
$\begin{array}{ll}\text { Research Square } & \begin{array}{l}\text { Preprints are preliminary reports that have not undergone peer review. } \\ \text { They should not be considered conclusive, used to inform clinical practice, } \\ \text { or referenced by the media as validated information. }\end{array}\end{array}$
}

\section{Development and Assessment of The Quality of Life Instruments for Chronic Diseases-Gout(QLICD-GO) (V2.0)}

\author{
Qiongling Liu \\ Guangdong Medical University \\ Chonghua Wan \\ Guangdong Medical University \\ Xiaohua Xie \\ Guangdong Medical University \\ Xu Liu \\ Guangdong Medical University \\ Xingshan Zhang \\ Guangdong Medical University \\ Jiawei Rao \\ Guangdong Medical University \\ Xingjie Wang \\ Guangdong Medical University \\ Haiyan Pan ( $\square$ haiyan_pan@126.com) \\ Guangdong Medical University https://orcid.org/0000-0003-4795-180X
}

\section{Research article}

Keywords: Gout, Quality of Life, Instrument, Reliability, Validity, Responsiveness

Posted Date: December 16th, 2020

DOI: https://doi.org/10.21203/rs.3.rs-124191/v1

License: (9) (i) This work is licensed under a Creative Commons Attribution 4.0 International License. Read Full License 


\section{Abstract}

Background: To develop and assess the Quality of Life Instruments for Chronic Diseases-Gout (QLICD-GO [V2.0]).

Methods: The instrument was developed using a programmatic decision-making method to combine the general module of the Quality of Life Instruments for Chronic Diseases and a new specific module. The instrument was assessed by measuring the quality of life of 116 patients with gout.

Results: The QLICD-GO(V2.0) included 28 items from the general module of chronic diseases and 12 items in three facets from the specific module. In addition to the field of physiological function, the internal consistency reliability of other fields and dimensions of the instrument was $>0.7$ and the split-half reliability was $>0.5$. Three common factors were extracted from the specific module, with a cumulative variance contribution rate of $57.54 \%$. The standardized response means of the specific module and the whole instrument were 0.94 and 1.20 , respectively.

Conclusions: The QLICD-GO(V2.0) has good reliability, validity, and responsiveness. The instrument comprehensively and objectively reflects the quality of life of patients with gout, and it can be used to assess treatment regimens developed by medical staff.

\section{Background}

In recent years, despite the variations in reports across countries, there has been a clear upward trend in the incidence of gout [1]. Gout is a chronic disease caused by an elevation in the serum urate level [2]. In Western Europe, 1-2\% of adults have been affected by gout, while the proportions have reached $4 \%$ in developed countries such as the United States and the United Kingdom [3]. Male patients outnumber female patients at a ratio of approximately 3:1. The prevalence of gout in China is about $1.1 \%$, which is increasing year by year. The age of gout is also becoming younger [4]. The prevalence of gout is increasing with socioeconomic development and changes in diet structure [5]. This system includes a general module (QLICD-GM, V1.0), which can be used with all types of chronic disease patients, and specific modules for different diseases, with each module being used for only the relevant disease[6]. Based on the Quality of Life Instruments for Chronic Diseases (QLICD-GM, V1.0) in China developed by Wan et al., the Quality of Life Instruments for Chronic Diseases-Gout (QLICD-GO, V2.0) was formulated and used to assess patients with gout during clinic visits between October 2015 and December 2016. The results are reported below.

\section{Study Subjects And Methods}

\subsection{Subjects}

The subjects were 161 patients with a confirmed diagnosis of gout in the Department of Rheumatology and Immunology in the First Affiliated Hospital of Shenzhen University (Shenzhen Second People's Hospital) and the Affiliated Hospital of Guangdong Medical University between October 2015 and December 2016. The diagnostic criteria were based on the 2015 American College of Rheumatology/European League Against Rheumatism Gout Classification Criteria [7]. The eligible patients had basic literacy skills. Pre- and posttreatment quality of life (QOL) assessments were performed after informed consent was obtained.

\subsection{Methods}

1.2.1 Instrument development The QLICD-GO(V2.0) was developed by combining the widely recognized general module of the Quality of Life Instruments for Chronic Diseases (QLICD-GM) with a specific module for gout. This general module, consisting of 28 items in three domains (physical function, psychological function, and social function), has previously been formulated on the basis of topic discussion, interviews with and investigations in patients with different chronic diseases and medical staff, and multiple discussions, analyses, screening, and revisions by research team members.

With reference to relevant literature and QOL instruments (universal and specific instruments) for patients with gout from China and abroad and in combination with clinical expertise on gout, we proposed a pool of 34 health-related QOL items specifically for patients with gout, covering gout-induced impacts on the physical, psychological, and social functioning of patients, and drug-related side effects. Each potential item for the gout-specific module was analyzed at meetings of the topic panel and core panel. Thirteen items for the specific module were selected and were organized into a patient questionnaire and a medical staff questionnaire. Based on five methods to screen items and the opinions of clinical experts, a 12-item gout-specific module was formed.

The QLICD-GO(V2.0) is divided into four aspects, including physical symptoms and signs (six items), functional limitation to daily life (one item), common drug-related side effects (two items), and unique psychological effects (three items). A five-point Likert scoring method is 
used, with possible scores between 1 and 5 or between -5 and -1 . The higher the score of a positive item, the better the QOL; the greater the absolute value of the score of a negative item, the worse the QOL. For positive items, there is no need to convert, the original score is the item score, for reverse items, it is necessary to "forward transform", that is, subtract the original score from 6 to get the item score.

1.2.2 Data collection Between October 2015 and December 2016, the investigator briefly explained the purpose of the survey and distributed the instrument to the patients, who filled in the instrument independently according to their actual conditions in the Rheumatic immune clinic and rheumatic immune Department of Affiliated Hospital of Guangdong Medical University. Because most patients with gout were outpatients, the subjects fill out the instrument on the day of initial consultation and on the day of subsequent consultation. Inclusion criteria: \gout was diagnosed according to the 2015 American rheumatology society / European anti rheumatism alliance gout classification standard [8]. खPrimary school and above education level, clear awareness can fill in the questionnaire. खNo mental illness or consciousness disorder. $₫$ No exclusion criteria. Exclusion criteria: $\otimes$ The patient had joint pain and dysfunction caused by other rheumatic diseases. $\otimes$ Combined with hypertension, diabetes and other chronic history and cancer history. \lt is associated with heart, liver, lung, kidney and other visceral diseases. खllliterate, delirious, mentally ill, critically ill, unwilling to cooperate.

1.2.3 Instrument assessment After conversion of item scores, the raw score (RS) in each aspect was calculated and converted to a standard score (SS) according to the range method to facilitate comparison between items. The SS of a domain was obtained by subtracting the lowest possible score of the domain $\left(S_{\min }\right)$ from the RS of the domain, the difference was divided by the range of the RS of the domain, and the result was multiplied by 100 . The calculation formula is $S S=\left(R S-S_{\min }\right) /\left(S_{\max }-S_{\min }\right) \times 100$. 3). The assessment covered the reliability (internal consistency reliability and split-half reliability), validity (content validity, construct validity, and criterion validity), and responsiveness (comparison of pre- and posttreatment mean scores, standardized response mean [SRM]) of the instrument. In this study, we use SF-36 as the calibration standard, because the calibration validity is lack of gold standard, and SF-36 is a widely recognized tool for measuring QOL [9].

\subsection{Statistical analysis}

SPSS 21.0 statistical software was used for data entry and analysis. The statistical methods included the following two categories: methods for instrument item screening (coefficient of variation, correlation analysis, and factor analysis) and methods for instrument assessment (paired $t$-test and correlation analysis).

\subsection{Patient and Public Involvement}

In this experiment, QLICD-GO(V2.0) was developed by combining the common module of chronic diseases with the specific module of gout. The common module of chronic diseases is now mature. We need to develop a specific module of gout. It is divided into issue group and core group, mainly composed of life quality researchers, rheumatology immunologists, epidemiologists, public health scholars, sociologists and other personnel at all levels. The selection of the main items of the topic group, the later development and evaluation of the main scale of the core group, finally formed the specific module of the gout scale.

The scale is patient self-rated. The researcher made a brief introduction to the questionnaire to the respondents. Under the condition of the patients' willingness, the questionnaire was distributed to the patients, and then it was taken back after the patients completed the questionnaire, and carefully checked whether there was any missing item. If there was any missing item, the researcher reminded the respondents to supplement it. If it still could not be filled in, it was treated as the default value and recorded the reason. Since the majority of gout patients are outpatients, in principle, the subjects fill in a questionnaire on the day of inquiry and the day of follow-up.

\section{Results}

\subsection{General information}

A total of 116 outpatients (aged $46.81 \pm 16.38$ years) with gout were investigated in this study. The details are presented in Table 1 .

\section{Table 1 General information of 116 patients with gout}




\begin{tabular}{|c|c|c|c|c|c|}
\hline Category & $\begin{array}{l}\text { Number of } \\
\text { patients }(\mathrm{N})\end{array}$ & $\begin{array}{l}\text { Constituent } \\
\text { ratio } \\
(\%)\end{array}$ & Category & $\begin{array}{l}\text { Number of } \\
\text { patients }(\mathrm{N})\end{array}$ & $\begin{array}{l}\text { Constituent } \\
\text { ratio } \\
(\%)\end{array}$ \\
\hline Sex & & & Nationality & & \\
\hline Male & 110 & 94.8 & Han & 116 & 100 \\
\hline Female & 6 & 5.2 & Others & 0 & 0 \\
\hline Educational level & & & Occupation & & \\
\hline Primary school & 8 & 6.9 & Worker & 33 & 28.4 \\
\hline Junior high school & 35 & 30.2 & Farmer & 26 & 22.4 \\
\hline $\begin{array}{l}\text { High school or technical } \\
\text { secondary school }\end{array}$ & 39 & 33.6 & Teacher & 6 & 5.2 \\
\hline \multirow[t]{2}{*}{ Junior college } & 19 & 16.4 & Cadre & 9 & 7.8 \\
\hline & & & Others & 29 & 25.0 \\
\hline Health insurance & & & $\begin{array}{l}\text { Household financial } \\
\text { status }\end{array}$ & & \\
\hline Self-paying & 109 & 94.0 & Poor & 8 & 6.9 \\
\hline Social health insurance & 7 & 6.0 & Fair & 99 & 85.3 \\
\hline Commercial health insurance & 0 & 0 & Good & 9 & 7.8 \\
\hline $\begin{array}{l}\text { Rural Cooperative health } \\
\text { insurance }\end{array}$ & 0 & 0 & & & \\
\hline Marital status & & & Clinical stage & & \\
\hline Unmarried & 19 & 16.4 & $\begin{array}{l}\text { Intercritical stage (with } \\
\text { tophi) }\end{array}$ & 2 & 1.7 \\
\hline Married & 95 & 81.9 & Acute stage & 86 & 74.10 \\
\hline Divorced & 1 & 0.9 & $\begin{array}{l}\text { Acute stage (with } \\
\text { tophi) }\end{array}$ & 23 & 19.80 \\
\hline Widowed & 1 & 0.9 & $\begin{array}{l}\text { Chronic stage (with } \\
\text { tophi) }\end{array}$ & 5 & 4.30 \\
\hline Age & $46.81 \pm 16.38$ & & & & \\
\hline
\end{tabular}

\subsection{Reliability analysis}

A total of 116 patients with gout were investigated using the QLICD-GO(V2.0) to analyze the internal consistency and split-half reliability of the general domain and specific domain. The Cronbach alpha coefficient of QLICD-GO(V2.0) in all fields is above 0.7 except for the physiological function field. The Cronbach's a coefficients of the general domain, specific domain, and whole instrument were $0.84,0.81$, and 0.55 , respectively; the split-half reliability coefficients of domains in the QLICD-GO(V2.0) were between 0.33 and 0.76 . The split-half reliability coefficients of the general domain, specific domain, and whole instrument were $0.73,0.65$, and 0.89 , respectively. Both coefficients showed relatively high reliability and good internal consistency of the instrument (see Table 2 for details).

Table 2 Internal consistency reliability of the QLICD-GO 


\begin{tabular}{|llll|}
\hline Domain & Number of items & Cronbach's a coefficient & Split-half reliability \\
\hline Specific domain (SPD) & 12 & 0.81 & 0.65 \\
\hline General domain (CGD) & 28 & 0.84 & 0.73 \\
\hline Physical domain (PHD) & 9 & 0.49 & 0.33 \\
\hline Psychological domain (PSD) & 11 & 0.79 & 0.76 \\
\hline Social domain (SOD) & 8 & 0.77 & 0.72 \\
\hline Total (TOT) & 40 & 0.55 & 0.89 \\
\hline
\end{tabular}

\subsection{Validity analysis}

2.3.1 Content validity The QLICD-GO(V2.0) has been developed in a stringent and programmatic manner under the QLICD system. The items of the instrument include dimensions and assessment contents required by the World Health Organization (WHO) and conforming to the definitions of health and QOL proposed by the WHO. These items cover the physical, psychological, and social status specific to patients with gout. The relatively good content validity of the QLICD-GO(V2.0) is justified on the basis of thorough discussion and screening by the expert panel.

2.3.2 Construct validity Since assessment of the construct validity of the general module of the instrument has been completed at an early stage, only the construct validity of the specific module was assessed in this section, mainly using exploratory factor analysis and itemdimension correlation analysis.

Exploratory factor analysis: Bartlett's test of sphericity of the specific module of the instrument yielded a $\chi^{2}$ value of $471.93(P<0.001)$, indicating that the variables were not independent and were suitable for factor analysis. In addition, the Kaiser-Meyer-Olkin (KMO) measure of sampling adequacy was 0.76 in this study, indicating that factor analysis was suitable (factor analysis is not suitable when $\mathrm{KMO}$ is $<0.5$ ). The principal component analysis method was used to extract common factors. According to the above discussion and eigenvalue $>1$, three common factors were extracted for the instrument after varimax rotation. The cumulative variance contribution rate was $57.54 \%$. Factor I included G07 and G010-G012 and mainly reflected the psychological changes in patients with gout; Factor II included G01-G04 and mainly reflected the clinical symptoms and signs in patients with gout; Factor III included G05, G06, G08, and G09, and it mainly reflected the persistent conditions and long-term drug-related side effects in patients with gout. The contribution rates of the three factors were $34.79 \%, 11.89 \%$, and $10.86 \%$, respectively. Among them, Factor I had the highest contribution rate. Broad consistency between the items reflected by these factors and the results obtained after screening and discussion of specific module items in the instrument indicates relatively good construct validity of the specific module items. The specific module factors and the factor loading coefficients of items are presented in Table 3 and Figure1.

Table 3 Specific module factors and factor loading coefficients for items $(\mathrm{N}=116)$ 


\begin{tabular}{|c|c|c|c|c|}
\hline \multirow[t]{2}{*}{ Item } & \multirow[t]{2}{*}{ Brief description } & \multicolumn{3}{|c|}{$\begin{array}{l}\text { Principal component } \\
\text { (variance contribution \%) }\end{array}$} \\
\hline & & $1(34.79 \%)$ & $2(11.89 \%)$ & 3 (10.86\%) \\
\hline GO 1 & Acute joint pain & 0.176 & 0.727 & 0.237 \\
\hline GO 2 & Joint redness and swelling & 0.148 & 0.870 & 0.064 \\
\hline GO 3 & Pyrexia & 0.230 & 0.550 & -0.183 \\
\hline GO 4 & Recurrent articular gout & 0.241 & 0.650 & 0.392 \\
\hline GO 5 & Tophi & -0.283 & 0.213 & 0.602 \\
\hline GO 6 & Nocturia & 0.157 & 0.160 & 0.770 \\
\hline GO 7 & Impact of lifestyle changes & 0.602 & 0.168 & -0.017 \\
\hline GO 8 & Drug-induced diarrhea & 0.414 & -0.089 & 0.431 \\
\hline GO 9 & Drug-induced rash and pruritus & 0.480 & -0.145 & 0.462 \\
\hline GO 10 & Anxiety about physical disability & 0.756 & 0.313 & 0.121 \\
\hline GO 11 & Anxiety about gout being passed on to your offspring & 0.787 & 0.279 & 0.039 \\
\hline GO 12 & Anxiety about long-term drug-related side effects & 0.788 & 0.351 & 0.057 \\
\hline
\end{tabular}

Item-dimension correlation: Coefficients of correlation between each item of the QLICD-GO (V2.0) and the scores of the general module's physical, psychological, and social domains and the gout-specific module were calculated. Correlation analysis showed relatively large coefficients of correlation between most items and their domains but small coefficients of correlation between these items and other domains, and the $r$ values were mostly above 0.4 . For example, the items of specific module unsurprisingly had closer correlations with the specific module areas than with the other three areas. It can be concluded that there were good correlations between QLICD-GO(V2.0) items and their dimensions and therefore satisfactory construct validity (see Table 4 for details).

\section{Table 4 Correlations between QLICD-GO items and their own domains}




\begin{tabular}{|c|c|c|c|c|}
\hline Item & Physical function & Psychological function & Social function & Specific module \\
\hline GPH1 & $0.534^{\star \star}$ & 0.175 & $0.188^{*}$ & 0.137 \\
\hline $\mathrm{GPH} 2$ & $0.332^{\star \star}$ & 0.031 & 0.129 & 0.161 \\
\hline GPH3 & $0.431^{\star \star}$ & $0.271^{\star \star}$ & $0.083^{*}$ & $0.218^{\star}$ \\
\hline GPH4 & $0.436^{\star *}$ & 0.012 & $0.263^{\star \star}$ & $0.279 * *$ \\
\hline GPH5 & 0.133 & $0.285^{\star \star}$ & 0.145 & $0.407 * \star$ \\
\hline GPH6 & $0.494^{\star \star}$ & 0.067 & 0.027 & $0.223^{\star}$ \\
\hline GPH7 & $0.573^{\star \star}$ & 0.007 & 0.014 & $0.354^{\star \star}$ \\
\hline GPH8 & $0.514^{\star \star}$ & 0.025 & 0.057 & $0.230 *$ \\
\hline GPH9 & $0.318^{\star \star}$ & $0.356^{\star \star}$ & 0.054 & $0.226^{\star}$ \\
\hline GPH10 & $0.537 \star \star$ & $0.396^{\star \star}$ & $0.208^{*}$ & $0.407 * \star$ \\
\hline GPS1 & $0.337^{\star \star}$ & 0.085 & $0.289 * \star$ & $0.311^{\star *}$ \\
\hline GPS2 & 0.167 & $0.402^{\star \star}$ & 0.032 & 0.163 \\
\hline GPS3 & $0.258^{\star \star}$ & 0.073 & $0.201^{\star}$ & 0.156 \\
\hline GPS4 & $0.237^{*}$ & $0.560 * \star$ & 0.118 & $0.260 * \star$ \\
\hline GPS5 & 0.091 & $0.643^{\star *}$ & 0.105 & $0.378^{* *}$ \\
\hline GPS6 & 0.041 & $0.633^{\star \star}$ & 0.192 & $0.492^{\star \star}$ \\
\hline GPS7 & 0.084 & $0.742^{\star \star}$ & $0.127 \star \star$ & $0.397 * \star$ \\
\hline GPS8 & 0.046 & $0.612^{\star \star}$ & 0.074 & 0.360 ** \\
\hline GPS9 & 0.146 & $0.671^{\star \star}$ & 0.174 & $0.477^{\star \star}$ \\
\hline GPS10 & 0.260 ** & 0.041 & 0.374 * & $0.234^{\star}$ \\
\hline GPS11 & 0.115 & $0.555^{\star \star}$ & 0.016 & $0.363^{\star \star}$ \\
\hline GS01 & $0.214^{\star \star}$ & 0082 & $0.605^{\star \star}$ & $0.220^{\star}$ \\
\hline GSO2 & 0.151 & 0.095 & $0.492^{\star \star}$ & 0.169 \\
\hline GSO3 & $0.195^{\star}$ & 0.147 & $0.482^{\star \star}$ & 0.052 \\
\hline GSO4 & 0.084 & 0.005 & $0.671^{\star \star}$ & 0.036 \\
\hline GSO5 & 0.109 & 0.020 & $0.657 \star \star$ & 0.080 \\
\hline GSO6 & 0.027 & $0.385^{\star \star}$ & 0.132 & $0.368^{\star *}$ \\
\hline GSO7 & $0.246^{\star \star}$ & $0.376^{\star \star}$ & 0.350 ** & 0.460 ** \\
\hline GSO8 & $0.183^{*}$ & 0.076 & $0.569 \star \star$ & 0.125 \\
\hline G01 & 0.138 & 0.320 ** & $0.216^{\star \star}$ & $0.667^{\star \star}$ \\
\hline G02 & $0.189 *$ & $0.246^{\star \star}$ & 0.122 & $0.455^{\star \star}$ \\
\hline GO3 & 0.177 & 0.290 ** & 0.075 & $0.691^{\star *}$ \\
\hline GO4 & 0.067 & 0.043 & 0.162 & 0.125 \\
\hline G05 & 0.105 & $0.239 \star \star$ & 0.045 & $0.509 * \star$ \\
\hline G06 & 0.049 & $0.319 * \star$ & 0.015 & $0.511^{\star *}$ \\
\hline G07 & 0.024 & 0.127 & 0.091 & $0.381^{\star *}$ \\
\hline G08 & 0.013 & $0.007^{\star}$ & $0.241^{*}$ & $0.368^{\star \star}$ \\
\hline G09 & 0.028 & $0.425^{\star \star}$ & 0.083 & $0.741^{\star *}$ \\
\hline
\end{tabular}

Page 7/14 


\begin{tabular}{|lllll|}
\hline G010 & 0.020 & $0.358^{\star \star}$ & 0.079 & $0.704^{\star \star}$ \\
\hline G011 & 0.008 & $0.402^{\star \star}$ & 0.152 & $0.774^{\star *}$ \\
\hline G012 & 0.098 & $0.194^{\star}$ & 0.077 & $0.637^{\star \star}$ \\
\hline
\end{tabular}

Note: **: $P<0.01, *: P<0.05$

GPHGout instrument physical function question, GPS Gout instrument psychological function question, GSO Gout instrument social function question, GO Gout instrument targeted question

2.3.3 Criterion validity The role function and the emotional function domain of the SF-36 instrument were weakly correlated with domains of the QLICD-GO(V2.0) (coefficients of correlation <0.4). For the remaining six domains of the SF-36 instrument, the coefficients of correlation with corresponding domains of the QLICD-GO(V2.0) were all $>0.4$. The coefficients of correlation between the corresponding domains were statistically significant $(P<0.05$; Table 5). The QLICD-GO(V2.0) therefore has relatively good criterion validity.

Table 5 Coefficients of correlation between domains of the QLICD-GO and those of the SF-36 instrument

\begin{tabular}{|c|c|c|c|c|}
\hline \multirow[t]{2}{*}{ SF-36 } & \multicolumn{4}{|c|}{ QLICD-GO } \\
\hline & PHD & PSD & SOD & SPD \\
\hline Physical function (PF) & $0.520 \star \star$ & $0.205^{\star}$ & $0.289 * \star$ & $0.398^{\star *}$ \\
\hline Role function (RF) & 0.356 ** & $0.267 \star \star$ & $0.317 \star \star$ & $0.379 * \star$ \\
\hline Bodily pain (BP) & $0.436^{\star \star}$ & $0.298^{\star \star}$ & $0.215^{\star}$ & $0.390 * *$ \\
\hline General health $(\mathrm{GH})$ & $0.255^{\star \star}$ & $0.399 * \star$ & $0.406 * \star$ & $0.441^{* \star}$ \\
\hline Vitality (VT) & $0.472^{\star \star}$ & $0.387^{\star \star}$ & $0.483^{\star \star}$ & $0.542^{\star \star}$ \\
\hline Social function (SF) & $0.298^{\star \star}$ & $0.363^{\star \star}$ & $0.371^{\star \star}$ & $0.426 * \star$ \\
\hline Role emotional (RE) & $0.321 * \star$ & $0.219 *$ & $0.318^{\star \star}$ & $0.342^{\star \star}$ \\
\hline Mental health $(\mathrm{MH})$ & $0.420 \star \star$ & $0.447 * \star$ & $0.485^{\star \star}$ & $0.554^{\star \star}$ \\
\hline
\end{tabular}

Note: ${ }^{*} P<0.05, * \star P<0.01$

\subsection{Responsiveness analysis}

To evaluate the responsiveness of the QLICD-GO (V2.0), differences between pre- and posttreatment scores were calculated for each domain, the general module, and the whole instrument using the paired $t$-test, and the SRM was also calculated. The SRM is defined as the absolute ratio of the mean difference between pre- and posttreatment values to the standard deviation of the difference between pre- and posttreatment values. The analysis showed significant differences between pre- and posttreatment scores for the domains, the general module, the specific module, and the whole instrument $(P<0.05)$. The SRMs were above 0.90 for the whole instrument and all domains except psychological function and social function, indicating that the QLICD-GO(V2.0) has favorable responsiveness and is sensitive in reflecting changes in the QOL of patients with gout. The details are presented in Table 6 and Figure 2.

Table 6 Responsiveness of the QLICD-GO 


\begin{tabular}{|c|c|c|c|c|c|}
\hline \multirow[t]{2}{*}{ Domain/Aspect } & \multirow{2}{*}{$\begin{array}{l}\text { Pretreatment } \\
\qquad \bar{\chi} \pm s\end{array}$} & \multirow{2}{*}{$\begin{array}{l}\text { Posttreatment } \\
\qquad \bar{\chi} \pm s\end{array}$} & \multicolumn{2}{|c|}{ Paired $t$-test } & \multirow[t]{2}{*}{ SRM } \\
\hline & & & $t$ & $P$ & \\
\hline Physical function (PHD) & $55.41 \pm 12.82$ & $71.92 \pm 11.51$ & -15.98 & $<0.001$ & 1.48 \\
\hline Basic physical function (BPF) & $59.09 \pm 15.04$ & $67.16 \pm 13.32$ & -6.82 & $<0.001$ & 0.63 \\
\hline Independence (IND) & $59.34 \pm 20.24$ & $83.69 \pm 18.00$ & -12.32 & $<0.001$ & 1.14 \\
\hline Energy and discomfort (EAD) & $40.30 \pm 20.86$ & $66.16 \pm 16.49$ & -13.37 & $<0.001$ & 1.24 \\
\hline Psychological function (PSD) & $61.79 \pm 16.23$ & $68.03 \pm 14.08$ & -5.52 & $<0.001$ & 0.51 \\
\hline Cognition (COG) & $63.69 \pm 18.50$ & $69.61 \pm 18.81$ & -3.28 & 0.001 & 0.30 \\
\hline Emotion (EMO) & $58.74 \pm 19.67$ & $65.73 \pm 16.37$ & -5.42 & $<0.001$ & 0.50 \\
\hline Will (WIL) & $74.35 \pm 23.65$ & $77.80 \pm 21.31$ & -1.91 & 0.059 & 0.18 \\
\hline Social function (SOD) & $75.54 \pm 14.91$ & $78.29 \pm 12.51$ & -3.03 & 0.003 & 0.28 \\
\hline Interpersonal communication (INC) & $81.47 \pm 15.85$ & $85.42 \pm 13.04$ & -4.03 & $<0.001$ & 0.37 \\
\hline Social support (SSS) & $76.22 \pm 17.66$ & $76.29 \pm 14.44$ & -0.05 & 0.958 & 0.00 \\
\hline Social role (SOR) & $65.63 \pm 22.35$ & $70.58 \pm 19.03$ & -2.84 & 0.005 & 0.26 \\
\hline General domain (CGD) & $63.38 \pm 11.92$ & $72.20 \pm 10.94$ & -11.60 & $<0.001$ & 1.08 \\
\hline Specific domain (SPD) & $52.89 \pm 17.74$ & $64.62 \pm 14.71$ & -10.08 & $<0.001$ & 0.94 \\
\hline Clinical symptoms (CLS) & $48.78 \pm 19.23$ & $74.21 \pm 15.88$ & -15.28 & $<0.001$ & 1.42 \\
\hline Drug-related side effects (DSE) & $77.59 \pm 21.84$ & $71.98 \pm 22.64$ & 2.92 & 0.004 & 0.27 \\
\hline Influence of special mentality life(EML) & $46.71 \pm 27.45$ & $46.55 \pm 25.94$ & 0.09 & 0.932 & 0.01 \\
\hline Total (TOT) & $60.31 \pm 12.27$ & $69.98 \pm 10.95$ & -12.95 & $<0.001$ & 1.20 \\
\hline
\end{tabular}

\section{Discussion}

The QLICD-GO (V2.0) has been developed for patients with gout based upon the foundation of the QLICD system, namely, the general module of the QLICD (QLICD-GM). The QLICD-GO(V2.0) reflects the clinical signs and symptoms, drug-related side effects, and psychological changes specific to patients with gout. The QLICD-GO (V2.0) consists of two parts, the mature general module and the specific module developed in this study, which includes 12 items (six items in clinical signs and symptoms, two items in drug-related side effects, and four items in unique psychological changes).

Item screening is one of the key steps in the development of any QOL instrument, which should adhere to the principles of high sensitivity, good representativeness, strong independence, sufficient certainty, and great significance [10]. Reliability refers to the repeatability or consistency of item scores [11]. Validity reflects the degree of conformity between the measured results of the instrument and the expected results [12]. Content validity refers to whether the selected items are capable of representing the characteristics of the measured content or subject [13]. Construct validity performs a factor analysis of all items based on the results of the survey, and classifies the items according to the loading of each item on each factor [14]. Responsiveness reflects the sensitivity to reflect changes in characteristic values of a subject [15]. Based on the assessment results, the QLICD-GO (V2.0) has good reliability, validity, and responsiveness. Zhang [16] has recommended 0.70 as the minimal Cronbach's a coefficient of internal consistency that would indicate relatively good internal consistency reliability. The Cronbach's a coefficient of the specific module was 0.81 , and the split-half reliability was 0.65 in this study, indicating relatively good reliability of the specific module. The validity of the instrument is relatively good based on content validity, construct validity, and criterion validity. The instrument is sensitive to changes in QOL of patients with gout before and after treatment. The responsiveness of an instrument is considered relatively good if absolute SRM is $>0.8$, moderate for an absolute SRM of about 0.5 , and relatively poor for an absolute SRM of about 0.2 [17]. Here the SRM was 0.94 for the specific module, and 1.2 for the whole instrument, indicating relatively good responsiveness of the QLICD-GO(V2.0). 
The QLICD-GO (V2.0) has been carefully developed according to an established scientific program, and it has shown good reliability, validity, and responsiveness in this initial assessment. It can objectively and comprehensively reflect the QOL of patients with gout and can be used by clinical staff to assess treatment regimens. The QLICD-GO (V2.0) was developed in the context of Chinese culture and provides a good basis and platform for measurement and assessment of the QOL of patients with gout in China.

\section{Strengths And Limitations Of This Study}

After many times of modification and investigation, the QLICD-GO(V2.0) has been investigated and modified on the basis of QLICD-GO (1.0), which has better reliability, validity, and responsiveness. QLICD-GO(V2.0) has the characteristics of Chinese cultural background, which provides a good foundation and platform for the measurement and evaluation of gout patients' quality of life in China. However, there are some shortcomings. The sample size of QLICD-GO(V2.0) is not large, which may affect the experimental results. QLICD-GO(V2.0) needs to be further validated, and the sample size needs to be expanded to provide more accurate results. Our sample objects are mainly from the outpatient department of the hospital. For patients who do not visit the outpatient department of the hospital, the applicability may not be strong. The longitudinal reactivity has not been tested in this study. In the long run, it is very important to see if QLICD-GO(V2.0) changes. These are also aspects that we need to actively modify in the future.

In general, QLICD-GO(V2.0) can be used as a useful tool to measure and evaluate the quality of life of Chinese speaking gout patients.

\section{Conclusion}

By enabling systematic measurement of the QOL of patients with gout both before and after treatment, the QLICD-GO (V2.0) may aid in public health education and patient-centered clinical research. In addition, more samples are needed to refine the instrument and more efforts are needed to further improve the QOL of patients with gout.

\section{Abbreviations}

QLICD-GO: the Quality of Life Instruments for Chronic Diseases-Gout

QLICD-GM: the Quality of Life Instruments for Chronic Diseases

QQL: quality of life

RS: raw score

SS: standard score

SRM: standardized response mean

SPD: Specific domain

GED: General domain

PHD: Physical domain

PSD: Psychological domain

SOD: Social domain

KMO: Kaiser-Meyer-Olkin

GPH: Gout instrument physical

GPS: Gout instrument psychological

GSO: Gout instrument social

TAR: Gout instrument targeted question

PF: Physical function

Page 10/14 
RF: Role function

BP: Bodily pain

GH: General health

SF: Social function

RE: Role emotional

$\mathrm{MH}$ : Mental health

BPF: Basic physical function

EAD: Energy and discomfort

SOD: Social function

INC: Interpersonal communication

SSS: Social support

SOR: Social role

SAS: Symptoms and signs

DSE: Drug-related side effects

PC: Psychological changes

\section{Declarations}

\section{Acknowledgments}

We thank all the participants for their contribution to this work.

\section{Author contributions}

QL $L$ and $X L$ contributed equally to this work. QL $L$ and $C H W$ designed the study. $X H X$ and XJ W did the literature search, study quality assessment and data extraction. JW R and XS Z performed the statistical analysis and drafted the tables and figures. X $L$ wrote the first draft of this analysis, and QL L helped to finish the final version. All authors approved the conclusions of our study.

\section{Ethics approval and consent to participate:}

After reviewing the ethics committee of the Guangdong Medical University Affiliated Hospital, the project is reasonably designed, advanced and practical. The study is in line with the declaration of Helsinki (WMA) and follows the standards of the ethics committee in conducting clinical studies. or this research, we have reached a consensus with the hospital and the hospital ethics committee. After the discussion of the ethics committee, the ethics committee agreed to adopt this research. Before we investigate the patient, we verbally explain the reason and purpose of the investigation to the patient. Patients verbally agreed that we would investigate them. We asked the patient's consent before we started the questionnaire. This step of verbally soliciting the patient's consent is in line with the requirements of the hospital ethics committee. The hospital ethics committee agreed with us. We have obtained the oral consent of the participants, and the ethics committee has approved this.

\section{Consent for publication}

Not applicable.

\section{Availability of data and material}

The datasets used and/or analyzed during the current study are available from the corresponding author on reasonable request. 


\section{Competing interests}

The authors declared that they have no conflicts of interest to this work.

\section{Authors' information}

QLL, Professor, Master Tutor, School of Nursing, Guangdong Medical University. XL, Master Degree Candidate, Qingdao West Coast New District People's Hospital. XHX, Shenzhen Second People's Hospital. CHW, Professor, Doctoral Supervisor, School of Humanities and Management, Guangdong Medical University. XSZ, Graduate Student of Guangdong Medical University. JWR, Graduate Student of Guangdong Medical University. XJW, Graduate Student of Guangdong Medical University. HYP,Associate Professor, Master Tutor, School of Public Health, Guangdong Medical University.

\section{Funding}

Supported by the National Natural Science Foundation of China $(71373058,8140277$, 30860248),Medical Science and Technology of Guangdong Province (B2019087), Young Innovative Talents Project of Guangdong Province (2018KQNCX096), Natural Science Foundation of Guangdong Province (2019A1515010875).

\section{References}

1. Li D, Zhang J. (2016). Research advances in modern epidemiology of gout and urate-lowering agents. Rheumatism and Arthritis, (4), $73-76$.

2. Li Z, Zhou Z, Hou X, et al. Replication of gout/urate concentrations GWAS susceptibility loci associated with gout in a Han Chinese population. Sci Rep. 2017;7(1):4094-100.

3. Tatlock S, Rudell K, Panter C, et al. What outcomes are important for gout patients? In-depth qualitative research into the gout patient experience to determine optimal endpoints for evaluating therapeutic interventions. Patient. 2016;10(1):65-79.

4. Rui L, Cheng H, Di W,et al. (2015). Prevalence of Hyperuricemia and Gout in Mainland China from 2000 to 2014::A Systematic Review and Meta-Analysis. Biomed Res Int,2015,762820.

5. Shufang Wang L, Feng M, Song, et al. Epidemiological survey of hyperuricemia and gout in newly recruited young male soldiers in Hohhot City. Medical Journal of the Chinese People's Armed Police Force. 2016;27(1):5-7 + 11.

6. Wan C, Tu X, Messing S, et al. Development and validation of the general module of the system of quality of life instruments for chronic diseases and its comparison with SF-36. Journal of pain symptom manage. 2010;42(1):-93-104.

7. Xuejun Zeng. An interpretation of the 2015 American College of Rheumatology/European League Against Rheumatism Gout Classification Criteria. Chinese Journal of Allergy Clinical Immunology. 2015;9(4):235-8.

8. Xuejun Zeng. Interpretation of 2015 American rheumatology society / European anti rheumatism alliance gout classification standard. Chinese Journal of clinical immunology and allergy, 2015, (04): 235-238.

9. Li F. Development and validation of the Quality of Life Instrument for Patients with Psoriasis (QLICD-PS V2.0) and its primary clinical applications, Guangdong Medical University, 2015.

10. Saimei Luo Q, Meng J, Luo, et al. Items selection on the specific module of Quality of Life Instruments for Patients with Prostate Cancer (QLICP-PR). Journal of Kunming Medical University. 2015;36(11):39-42.

11. Chonghua Wan H, Li X Fan, et al. Development and validation of the coronary heart disease scale under the system of quality of life instruments for chronic diseases QLICD-CHD: combinations of classical test theory and generalizability theory. Health Quality of Life Outcomes. 2014;12(1):82-93.

12. Annet F, Lenderink I, Zoer, Henk F, van der Molen, et al. Review on the validity of self-report to assess work-related diseases. Int Arch Occup Environ Health. 2012;85(3):229-51.

13. Xinping Huang. Development and application of the Quality of Life Instrument for Patients with Chronic Renal Failure (QLICD-CRF), Kunming Medical University, 2012.

14. Liuping Chen. Development and its applications of Quality of Life Instruments for Chronic Diseases: Pulmonary Tuberculosis (QLICDPT), Kunming Medical University, 2012.

15. Yanchun S, Yang Z, Wan C, et al. Development and validation of the pulmonary tuberculosis scale of the system of Quality of Life Instruments for Chronic Diseases (QLICD-PT). Health Quality of Life Outcomes. 2018;16(1):137-47.

16. Zhang Y. Feifei Zhou, \& Yu Sun. (2015). Assessment of health-related quality of life using the SF-36 in Chinese cervical spondylotic myelopathy patients after surgery and its consistency with neurological function assessment: a cohort study[J]. Health Quality of Life

Page $12 / 14$ 
Outcomes, 13(01):39-46.

17. Sun Z. Yongyong Xu. (2010). Medical Statistics, Third Edition. Beijing: People's Medical Publishing House, 3:435-439.

\section{Figures}

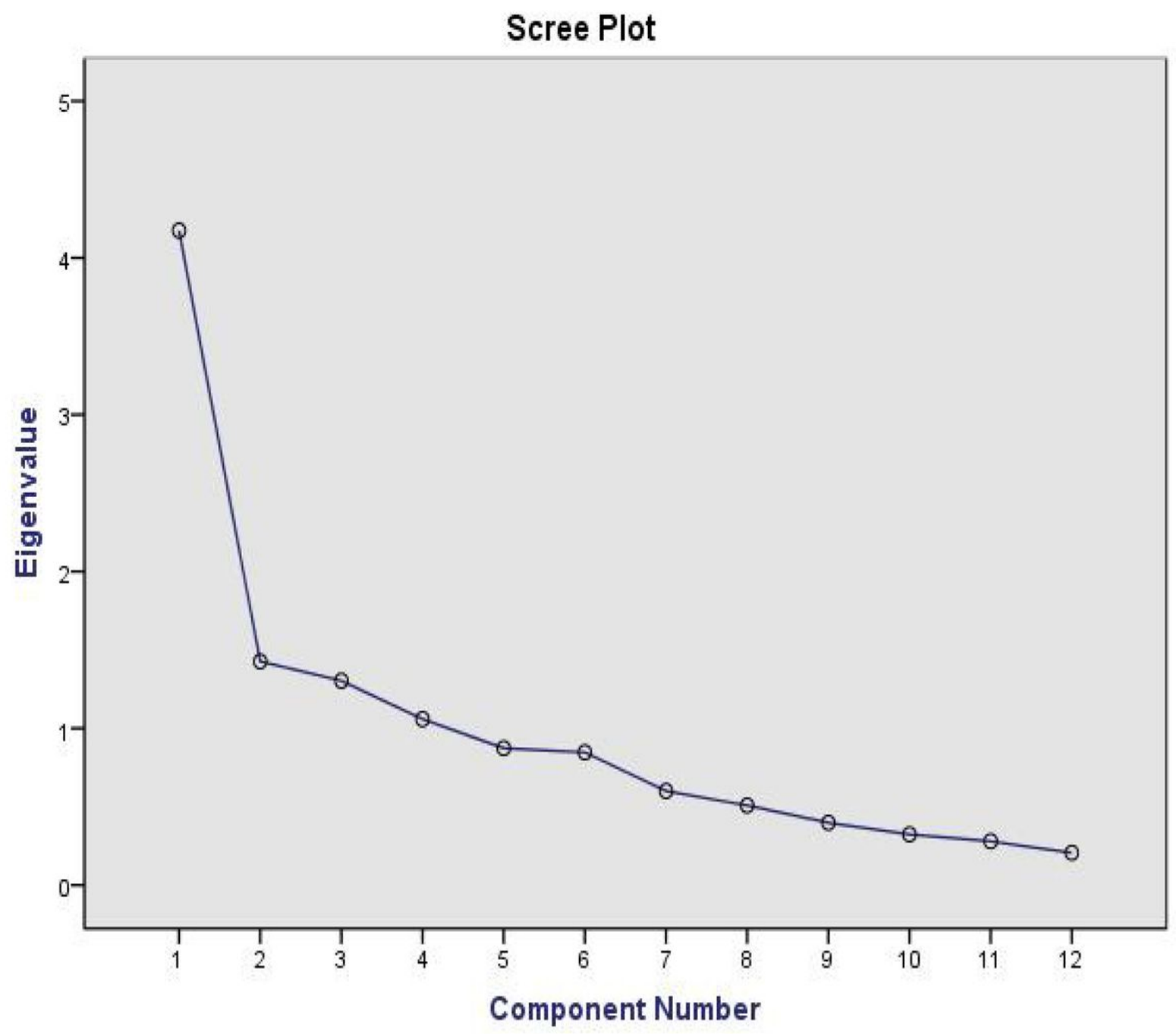

Figure 1

Scree plot of items in the specific module of QLICD-GO (V2.0) 


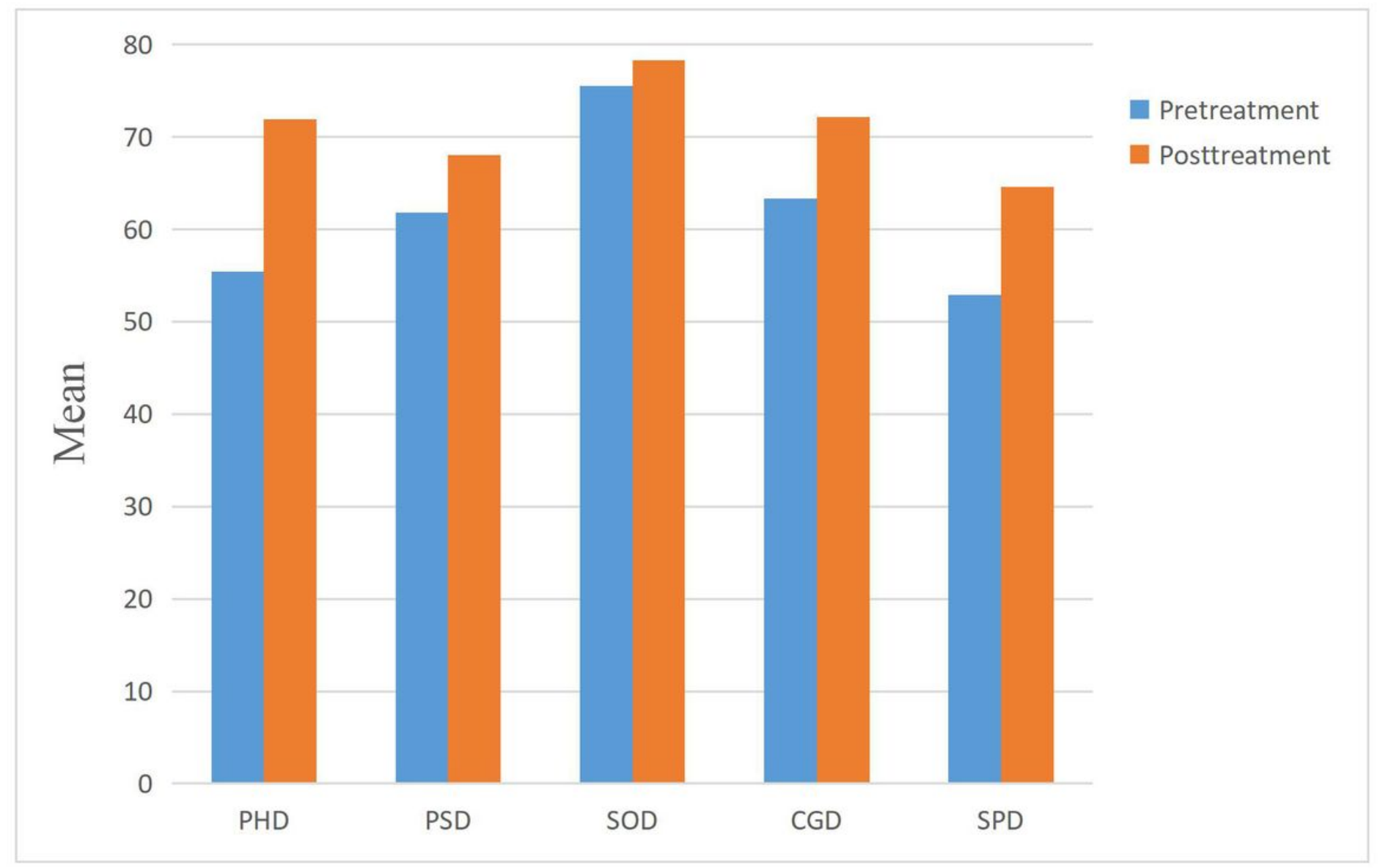

\section{Figure 2}

Mean scores in five domains of QLICD-GO (V2.0) pre- and posttreatment

\section{Supplementary Files}

This is a list of supplementary files associated with this preprint. Click to download.

- Questionnaire.docx 